\title{
Asymptotic and Exponential Stability of General Classes of Continuous-Time Power Control Laws in Wireless Networks
}

\author{
Hamid Reza Feyzmahdavian, Themistoklis Charalambous and Mikael Johansson
}

\begin{abstract}
This paper develops a comprehensive stability analysis framework for continuous-time power control algorithms in wireless networks under bounded time-varying communication delays. Our first set of results establish global asymptotic stability of power control laws involving two-sided scalable interference functions, and include earlier work on standard interference functions as a special case. We then consider contractive interference functions and demonstrate that the associated continuous-time power control laws always have unique fixed points, which are exponentially stable even in the presence of bounded heterogeneous time-varying delays. For this class of interference functions, we derive an explicit bound on the decay rate that allows us to quantify the impact of delays on the convergence time of the algorithm. Numerical simulations illustrate our theoretical results.
\end{abstract}

\section{INTRODUCTION}

The seminal work by Foschini and Miljanic [1] on continuous- and discrete-time power control triggered off numerous publications for both continuous-time power control (e.g., [2]-[6]) and discrete-time power control (e.g., [7][11]). An elegant axiomatic framework for studying a general class of discrete-time power control, the so-called standard interference functions, was proposed by Yates [7]. This framework includes the discrete-time Foschini-Miljanic (FM) algorithm [1] and several nonlinear power control algorithms as special cases. This framework was later extended by Sung and Leung [8] to a more general class, the so-called twosided scalable interference functions, to allow for simple and powerful analysis of certain opportunistic power control laws. While these frameworks are general and guarantee synchronous and asynchronous convergence of discrete-time power control algorithms in their class, the existence of fixed points has to be established separately, and there is no information about the convergence rate of the algorithms. Recently, a new framework, called contractive interference functions, was proposed by Feyzmahdavian et al. [11] that guarantees contractivity of the interference functions and hence unique fixed points, as well as linear convergence rates for discrete-time synchronous and asynchronous iterations.

While communication delays are inevitably omnipresent in networks, they have not been considered for continuoustime power control until recently. Charalambous et al. [4] using the multivariate Nyquist criterion showed that the continuous-time FM algorithm is asymptotically stable for arbitrary constant delays, while Zappavinga et al. [6] using

H. R. Feyzmahdavian, T. Charalambous, and M. Johansson are with the Automatlc Control Lab, ACCESS Linnaeus Center, School of Electrical Engineering, KTH-Royal Institute of Technology, SE-100 44 Stockholm, Sweden. Emails: \{hamidrez, themisc, mikaelj\}@kth.se. theory for positive systems proved that the FM algorithm is asymptotically stable even in the presence of bounded time-varying communication delays and topology changes. The continuous-time counterpart of the standard interference functions firstly appeared in [3] and it was later analysed in [5] using Lyapunov-Razumikhin functions.

In this paper, we consider general classes of continuoustime distributed power control algorithms, which are continuous-time versions of those proposed in [8] and [11]. We analyze the continuous-time two-sided scalable interference functions (for which standard interference functions constitute a special case) and contractive interference functions. Firstly, we prove that if there exists a feasible steady state power vector, power-control laws involving two-sided scalable interference functions are asymptotically stable for arbitrary bounded heterogeneous time-varying delays. Next, for the contractive interference functions, we prove that associated continuous-time power control algorithms converge exponentially to the unique fixed points, even in the presence of bounded communication delays. For this class of interference functions, we derive an explicit bound on the decay rate, thus quantifying the impact of delays on the convergence time of the algorithm. The validity of our theoretical results is demonstrated via an illustrative example for the continuous-time version of the Utility-Based Power Control (UBPC) algorithm proposed by Xiao et al. [12]

The remainder of the paper is organized as follows. In Section II, we introduce the notation that will be used throughout the paper and review some required preliminaries that are useful for the development of the results in this paper. Section III gives a description of the problem under consideration, while the main results of this paper are stated in Sections IV and V. An illustrative example is presented in Section VI, justifying the validity of our results. Finally, concluding remarks are given in Section VII.

\section{NOTATION AND PRELIMINARIES}

\section{A. Notation}

Vectors are written in bold lower case letters and matrices in capital letters. We have $\mathbb{R}, \mathbb{N}$, and $\mathbb{N}_{0}$ for the set of real numbers, natural numbers, and the set of natural numbers including zero, respectively. The non-negative orthant of the $n$-dimensional real space $\mathbb{R}^{n}$ is represented by $\mathbb{R}_{+}^{n}$. The $i^{t h}$ component of a vector $\boldsymbol{x} \in \mathbb{R}^{n}$ is denoted by $x_{i}$, and the notation $\boldsymbol{x} \geq \boldsymbol{y}$ implies that $x_{i} \geq y_{i}$ for all components $i$. For a matrix $A \in \mathbb{R}^{n \times n}, a_{i j}$ denotes the entry in row $i$ and column $j$. The spectral radius of a matrix $A$ is the largest 
magnitude of the eigenvalues of $A$ and is denoted by $\rho(A)$. Given a vector $\boldsymbol{v}>\mathbf{0}$, the weighted $l_{\infty}$ norm is defined by

$$
\|\boldsymbol{x}\|_{\infty}^{\boldsymbol{v}}=\max _{1 \leq i \leq n} \frac{\left|x_{i}\right|}{v_{i}} .
$$

The function $\operatorname{sgn}(x)$ is the signum function defined by $\operatorname{sgn}(x)=1$ if $x>0, \operatorname{sgn}(x)=-1$ if $x<0$, and $\operatorname{sgn}(0)=0$. For a given $\tau_{\max }>0, \mathcal{C}$ denotes the space of continuous functions mapping the interval $\left[-\tau_{\max }, 0\right]$ into $\mathbb{R}^{n}$. We also denote

$$
\mathcal{C}_{++}=\left\{\varphi(\cdot) \in \mathcal{C} \mid \varphi(\theta)>0, \theta \in\left[-\tau_{\max }, 0\right]\right\} .
$$

\section{B. Preliminaries}

Next, we review the key definitions and results necessary for developing the main results of this paper. The following definition introduce contraction mappings.

Definition 1 A mapping $f: \mathbb{R}^{n} \rightarrow \mathbb{R}^{n}$ is called a ccontraction if there exists a constant $c \in[0,1)$ such that

$$
\|\boldsymbol{f}(\boldsymbol{x})-\boldsymbol{f}(\boldsymbol{y})\| \leq c\|\boldsymbol{x}-\boldsymbol{y}\|, \quad \forall x, y \in \mathbb{R}^{n},
$$

where $\|\cdot\|$ is some norm on $\mathbb{R}^{n}$.

An attractive feature of contraction mappings is that they always have a unique fixed point.

Proposition 1 ( [13, Chapter 3]) If $f: \mathbb{R}^{n} \rightarrow \mathbb{R}^{n}$ is a $c$ contraction, then $f$ has a unique fixed point, i.e.,

$$
\exists \boldsymbol{x}^{\star} \in \mathbb{R}^{n} \text { such that } \boldsymbol{f}\left(\boldsymbol{x}^{\star}\right)=\boldsymbol{x}^{\star} .
$$

\section{Problem Statement}

We consider a wireless system consisting of $n$ mobile users communicating over the same frequency channel. The continuous-time power control algorithm, firstly considered in its general form by [3], is given by

$$
\frac{\mathrm{d} p_{i}(t)}{\mathrm{d} t}=k_{i}\left(-p_{i}(t)+I_{i}(\boldsymbol{p}(t))\right), \quad i=1, \ldots, n .
$$

Here, $p_{i}(t)$ is the transmitted power of user $i$ at time $t$, $I_{i}: \mathbb{R}_{+}^{n} \mapsto \mathbb{R}_{+}$is the interference function modeling the interference and noise experienced by the intended receiver of user $i$, and $k_{i}$ is a positive constant. The well-known continuous-time Foschini-Miljanic (FM) algorithm [1], for example, considers the linear interference function given by

$$
I_{i}(\boldsymbol{p})=\gamma_{i} \frac{\sum_{j \neq i} g_{i j} p_{j}+\eta_{i}}{g_{i i}}, \quad i=1, \ldots, n,
$$

where $g_{i j}$ is the channel gain on the link between user $j$ and the receiver of user $i, \gamma_{i}$ is the target Signal-to-Interferenceand-Noise Ratio (SINR) of user $i$, and $\eta_{i}$ is the background noise at the receiver of user $i$.

In practice, there will always be a signalling delay associated with transmitting the perceived interference at the transmitter to the receiver, so that it can adjust the power according to the power control law. Consequently, a realistic analysis of the continuous-time power control algorithm must consider heterogeneous time-varying delays. More precisely, the continuous-time power control algorithm (1), when the time delay is introduced becomes

$$
\begin{aligned}
\frac{\mathrm{d} p_{i}(t)}{\mathrm{d} t} & =k_{i}\left(-p_{i}(t)+I_{i}\left(\boldsymbol{p}^{d_{i}}(t)\right)\right), \quad t \geq 0, \\
p_{i}(t) & =\varphi_{i}(t), \quad t \in\left[-\tau_{\max }, 0\right], i=1, \ldots, n .
\end{aligned}
$$

Here, $\varphi(\cdot)=\left[\varphi_{1}(\cdot), \ldots, \varphi_{n}(\cdot)\right]^{T}$ is a continuous vector valued function specifying the initial condition of the system, and

$$
\boldsymbol{p}^{d_{i}}(t)=\left[p_{1}\left(t-\tau_{1}^{i}(t)\right), \ldots, p_{n}\left(t-\tau_{n}^{i}(t)\right)\right]^{T} .
$$

The delays $\tau_{j}^{i}(t)$ are assumed to be time-varying continuous functions with respect to $t$ and satisfy

$$
0 \leq \tau_{j}^{i}(t) \leq \tau_{\max }, \quad \forall i, j, t \geq 0,
$$

where $\tau_{\max }$ is a positive constant providing an upper bound on the maximum allowable delay. Moreover, no restriction on the derivative of $\tau_{j}^{i}(t)$ is imposed.

To put our work in context, note that the discrete-time analog of (3) consists of iterations of the type

$$
p_{i}(t+1)=I_{i}\left(\boldsymbol{p}^{d_{i}}(t)\right), \quad t \in \mathbb{N}_{0} .
$$

Such iterations have been studied under three frameworks; standard interference functions [7], two-sided scalable interference functions [8], and contractive interference functions [11]. Specifically, it was shown that if $\boldsymbol{I}(\boldsymbol{p})$ is a standard or two-sided scalable interference function, and a power vector $\boldsymbol{p}^{\star}$ satisfying $\boldsymbol{p}^{\star}=\boldsymbol{I}\left(\boldsymbol{p}^{\star}\right)$ exists, then the iterates generated by (4) converge asymptotically to $\boldsymbol{p}^{\star}[8$, Theorem 12]. If $\boldsymbol{I}(\boldsymbol{p})$ is contractive, then the iterates (4) converges to a unique fixed point at a linear rate [11, Theorem 9], i.e., the distance between the iterates and the fixed point decays exponentially.

The aim of this paper is to develop a stability analysis framework for the continuous-time power control algorithm (3) that is equally comprehensive as the theory for its discrete-time counterpart. Our main objectives are therefore to $(i)$ study the asymptotic stability of the continuous-time power control algorithm with heterogeneous time-varying delays described by (3) when the interference function $\boldsymbol{I}(\boldsymbol{p})$ is two-sided scalable; and to (ii) analyse the exponential stability of (3) when the interference function is contractive and determine how the convergence rate depends on the magnitude of the time delays.

\section{STANDARD AND Two-Sided SCALABLE INTERFERENCE FUNCTIONS}

As observed by Yates [7], many interference functions share important properties that allow them to be analyzed in a common framework. This observation led to the definition of standard interference functions.

Definition 2 A function $I: \mathbb{R}_{+}^{n} \rightarrow \mathbb{R}_{+}^{n}$ is called a standard interference function, if for all $\boldsymbol{p} \geq \mathbf{0}$ the following properties are satisfied.

- Positivity: $\boldsymbol{I}(\boldsymbol{p})>\mathbf{0}$, 
- Monotonicity: If $\boldsymbol{p} \geq \boldsymbol{p}^{\prime}$, then $\boldsymbol{I}(\boldsymbol{p}) \geq \boldsymbol{I}\left(\boldsymbol{p}^{\prime}\right)$,

- Scalability: For all $\alpha>1, \alpha \boldsymbol{I}(\boldsymbol{p})>\boldsymbol{I}(\alpha \boldsymbol{p})$.

Sung and Leung [8] introduced the following generalized class of interference functions, which are useful for analyzing certain classes of opportunistic power control laws.

Definition 3 A function $I: \mathbb{R}_{+}^{n} \rightarrow \mathbb{R}_{+}^{n}$ is called a two-sided scalable interference function, if for all $\boldsymbol{p} \geq \mathbf{0}, \boldsymbol{I}(\boldsymbol{p})$ satisfies

- Positivity: $\boldsymbol{I}(\boldsymbol{p})>\mathbf{0}$,

- Two-sided scalability: For all $\alpha>1$,

$$
\frac{1}{\alpha} \boldsymbol{p} \leq \boldsymbol{p}^{\prime} \leq \alpha \boldsymbol{p} \Rightarrow \frac{1}{\alpha} \boldsymbol{I}(\boldsymbol{p})<\boldsymbol{I}\left(\boldsymbol{p}^{\prime}\right)<\alpha \boldsymbol{I}(\boldsymbol{p}) .
$$

Even though the monotonicity and scalability conditions of standard interference functions have been replaced by the two-sided scalability condition, one can show that every standard interference function is also two-sided scalable [8, proposition 4]. However, the following example shows that the converse is, in general, not true. Consider the function

$$
I(p)= \begin{cases}\sqrt{p}, & 0<p \leq 1, \\ \frac{1}{\sqrt{p}}, & p>1,\end{cases}
$$

which is two-sided scalable. However, monotonicity property does not hold and, hence, the function is not standard.

The main properties of two-sided scalable interference functions can be summarized as follows.

Proposition 2 ( [8], [9]) Let I : $\mathbb{R}_{+}^{n} \rightarrow \mathbb{R}_{+}^{n}$ be a two-sided scalable interference function. Then,

(a) $\boldsymbol{I}(\boldsymbol{p})$ is continuous at $\boldsymbol{p}$ for all $\boldsymbol{p}>\mathbf{0}$.

(b) If $\boldsymbol{I}(\boldsymbol{p})$ has a fixed point, then that fixed point is unique.

(c) If $\boldsymbol{p} \neq \boldsymbol{p}^{\prime}$, then $d\left(\boldsymbol{I}(\boldsymbol{p}), \boldsymbol{I}\left(\boldsymbol{p}^{\prime}\right)\right)<d\left(\boldsymbol{p}, \boldsymbol{p}^{\prime}\right)$, where

$$
d\left(\boldsymbol{p}, \boldsymbol{p}^{\prime}\right)=\max _{i=1, \ldots, n}\left\{\max \left\{\frac{p_{i}}{p_{i}^{\prime}}, \frac{p_{i}^{\prime}}{p_{i}}\right\}\right\} .
$$

We begin by studying the asymptotic stability of the continuous-time power control algorithm (3) with $\boldsymbol{I}(\boldsymbol{p})$ being a two-sided scalable interference function. Thanks to $k_{i}$ being a positive constant and $I_{i}(\cdot)$ being positive, differential equation (3) defines a positive system ${ }^{1}$ [14, Chapter 3]. Therefore, the physical constraint that the power should be nonnegative $\left(p_{i}(t) \geq 0\right)$ is automatically fulfilled. The following theorem is our first key result, which shows that if a two-sided scalable interference function $\boldsymbol{I}(\boldsymbol{p})$ has a fixed point $\boldsymbol{p}^{\star}>\mathbf{0}$, then the solution to (3) converges asymptotically to $\boldsymbol{p}^{\star}$ for any initial condition $\varphi(\cdot) \in \mathcal{C}_{++}$.

Theorem 1 Suppose a two-sided scalable interference function $I: \mathbb{R}_{+}^{n} \rightarrow \mathbb{R}_{+}^{n}$ has a fixed point $\boldsymbol{p}^{\star}>\mathbf{0}$. Then, the continuous-time power control algorithm (3) is asymptotically stable for any initial condition $\varphi(\cdot) \in \mathcal{C}_{++}$and for any proportionality constant, $k_{i}>0$.

\footnotetext{
${ }^{1} \mathrm{~A}$ dynamical system is said to be positive if every trajectory of the system starting from nonnegative initial conditions remains forever in the positive orthant.
}

Proof: See Appendix A.

Remark 1 The delay independence of continuous-time power control algorithms with standard interference functions was previously considered in [5, Theorem 4], using a Lyapunov-Razumikhin approach. Since every standard interference function is also two-sided scalable, Theorem 1 recovers the delay independence of standard interference functions as a special case.

\section{CONTRaCtive Interference Functions}

Two-sided scalable interference functions do not necessarily have fixed points in the positive orthant (consider for example $I(p)=p+1$ ), and the existence of fixed points has to be verified separately. Furthermore, no guarantees about the convergence rate of (3) are given. In [11], contractive interference functions, a slight modification of the standard interference functions, were introduced to allow for a more powerful analysis of distributed power control algorithms.

Definition 4 A function $I: \mathbb{R}_{+}^{n} \rightarrow \mathbb{R}_{+}^{n}$ is said to be a $c$ contractive interference function if it, for all $\boldsymbol{p} \geq \mathbf{0}$, satisfies the following conditions

- Positivity: $\boldsymbol{I}(\boldsymbol{p})>\mathbf{0}$,

- Monotonicity: If $\boldsymbol{p} \geq \boldsymbol{p}^{\prime}$, then $\boldsymbol{I}(\boldsymbol{p}) \geq \boldsymbol{I}\left(\boldsymbol{p}^{\prime}\right)$,

- Contractivity: There exists a constant $c \in[0,1)$, and a vector $\boldsymbol{v}>\mathbf{0}$ such that for all $\epsilon>0$,

$$
\boldsymbol{I}(\boldsymbol{p}+\epsilon \boldsymbol{v}) \leq \boldsymbol{I}(\boldsymbol{p})+c \epsilon \boldsymbol{v} .
$$

Contrary to the result for standard and two-sided scalable interference functions, contractive interference functions always define contraction mappings in the weighted $l_{\infty}$ norm, hence $\boldsymbol{I}(\boldsymbol{p})=\boldsymbol{p}$ has a unique solution in $\mathbb{R}_{+}^{n}$ :

Proposition 3 ( [11]) If I : $\mathbb{R}_{+}^{n} \rightarrow \mathbb{R}_{+}^{n}$ is a c-contractive interference function, then it has a unique fixed point $p^{\star} \in \mathbb{R}_{+}^{n}$, and

$$
\left\|\boldsymbol{I}(\boldsymbol{p})-\boldsymbol{I}\left(\boldsymbol{p}^{\prime}\right)\right\|_{\infty}^{\boldsymbol{v}} \leq c\left\|\boldsymbol{p}-\boldsymbol{p}^{\prime}\right\|_{\infty}^{\boldsymbol{v}}, \quad \forall \boldsymbol{p}, \boldsymbol{p}^{\prime} \in \mathbb{R}^{n},
$$

where $c \in[0,1)$.

In [11], it was shown that the linear interference function (2), as well as many practical interference functions from the literature are contractive. In particular, all the examples provided in Yate's original paper [7] were shown to be contractive.

Throughout this section we will use the following concept of exponential stability.

Definition 5 The solution $\boldsymbol{p}(t)=\boldsymbol{p}^{\star}$ of (3) is said to be globally exponentially stable if there exist positive reals $\alpha$ and $\beta$ such that for every initial function $\varphi(\cdot)$, the solution $\boldsymbol{p}(t)$ of (3) satisfies

$\left\|\boldsymbol{p}(t)-\boldsymbol{p}^{\star}\right\| \leq \beta e^{-\alpha t}\left(\sup _{-\tau_{\max } \leq s \leq 0}\left\|\boldsymbol{\varphi}(s)-\boldsymbol{p}^{\star}\right\|\right), \quad \forall t \geq 0$.

Clearly, exponential stability implies asymptotic stability. 
We will now show that if the interference function is c-contractive, then the continuous-time power control law described by (3) converges exponentially to the unique fixed point. Moreover, an explicit bound on the convergence rate of (3) is provided that allows us to quantify the impact of the magnitude of the time delays on the convergence rate. Note that contractive interference functions are also positive and hence, the physical constraint that the power should be nonnegative $\left(p_{i}(t) \geq 0\right)$ is fulfilled.

Theorem 2 Suppose $I: \mathbb{R}_{+}^{n} \rightarrow \mathbb{R}_{+}^{n}$ is c-contractive. Then, the unique fixed point $\boldsymbol{p}^{\star}$ of the continuous-time power control algorithm (3) is exponentially stable for any initial condition $\varphi(\cdot) \in \mathcal{C}_{++}$and for any proportionality constant $k_{i}>0$. In particular, for all $t \geq 0$,

$\left\|\boldsymbol{p}(t)-\boldsymbol{p}^{\star}\right\|_{\infty}^{\boldsymbol{v}} \leq e^{-k_{\min }(1-\bar{c}) t}\left(\sup _{-\tau_{\max } \leq s \leq 0}\left\|\boldsymbol{\varphi}(s)-\boldsymbol{p}^{\star}\right\|_{\infty}^{\boldsymbol{v}}\right)$

where $k_{\min }=\min _{1 \leq i \leq n} k_{i}$, and $\bar{c}$ is the unique positive solution of the equation

$$
\bar{c}=c e^{k_{\min }(1-\bar{c}) \tau_{\max }} .
$$

Proof: See Appendix B.

Although Equation (6) does not admit an explicit solution, its solution $\bar{c}$ is always unique and satisfies

$$
c \leq \bar{c}<1 \text {. }
$$

Moreover, $\bar{c}$ is monotonically increasing with $\tau_{\max }$ and approaches one as $\tau_{\max }$ tends to infinity. Hence, while the power control law remains exponentially stable for arbitrary delays and proportionality constant, the convergence rate deteriorates with increasing delays. Furthermore, convergence rate is directly related to the convergence time of stable solutions of (3). Specifically, if we define the convergence time $T_{\delta}$ as the smallest $t$ such that $\left\|\boldsymbol{p}(t)-\boldsymbol{p}^{\star}\right\|_{\infty}^{\boldsymbol{v}} \leq \delta$, then, by Theorem 2 , we have

$$
R_{0} e^{-k_{\min }(1-\bar{c}) t} \leq \delta
$$

where

$$
R_{0}=\sup _{-\tau_{\max } \leq s \leq 0}\left\|\boldsymbol{\varphi}(s)-\boldsymbol{p}^{\star}\right\|_{\infty}^{\boldsymbol{v}}
$$

It follows that

$$
T_{\delta}=\frac{1}{k_{\min }(1-\bar{c})} \ln \frac{R_{0}}{\delta}
$$

We can see that the convergence time goes to $\infty$ as $\tau_{\max }$ tends to $\infty$.

\section{NUMERICAL EXAMPLES}

We consider a continuous-time implementation of the Utility-Based Power Control (UBPC) algorithm from [12], whose associated interference function is

$$
I_{i}^{u}(\mathbf{p})=\left(\frac{\sum_{j \neq i} g_{i j} p_{j}+\eta_{r}}{g_{i i}}\right) f_{i}^{-1}\left(\alpha_{i} \frac{\sum_{j \neq i} g_{i j} p_{j}+\eta_{r}}{g_{i i}}\right),
$$

where $i=1, \ldots, n$, and $\alpha_{i}$ is a price coefficient. Here, $f_{i}\left(S I R_{i}\right)=U_{i}^{\prime}\left(S I R_{i}\right)$ in the concave part of $U_{i}$ where $U_{i}$ is a utility function of user $i$. In their paper, Xiao et al. use a sigmoidal utility function

$$
U_{i}\left(S I R_{i}\right)=\frac{1}{1+e^{-a_{i}\left(S I R_{i}-b_{i}\right)}},
$$

where

$$
b_{i}=\gamma_{i}-a_{i}^{-1} \ln \left(a_{i} \gamma_{i}-1\right) .
$$

Let us define $M^{b}=\left[m_{i j}^{b}\right]$ to be

$$
m_{i j}^{b}=\left\{\begin{array}{cc}
b_{i} \frac{g_{i j}}{g_{i i}}, & j \neq i \\
0, & j=i .
\end{array}\right.
$$

In [11], it is shown that if $c=\rho\left(M^{b}\right)<1$, then $I^{u}$ is a c-contractive interference function. We will next show that Theorem 2 provides an explicit bound on the decay rate of UBPC algorithm that allows us to analytically quantify the impact of delays on the convergence rate of the algorithm.

Consider a wireless network with 4 users, characterized by matrix (9),

$$
M=\left[\begin{array}{cccc}
0 & 0.3558 & 0.0354 & 0.1737 \\
0.0522 & 0 & 0.0012 & 0.0229 \\
0.2169 & 1.2433 & 0 & 0.5503 \\
1.1938 & 0.8062 & 0.0206 & 0
\end{array}\right]
$$

The SINR threshold and the thermal noise for each node is set to $\gamma_{i}=1.5$ and $\eta_{i}=0.04 \mathrm{mWatts}$, respectively. The parameter $\alpha_{i}$ is set to 5000 . We assume that four users in the system use sigmoidal utility function with $a_{i}=1.33$, and $b_{i}$ is found according to (8). The spectral radius of the network is $\rho\left(M^{b}\right)=0.88297$, hence $c=0.88297$. The initial power $p_{i}(0)$ for all users is set to $1 \mathrm{mWatt}$. A simulation of the network characterized by the matrix (9) shows that SINRs converge to the desired SINR and power levels to the minimal power vector; see Figure 1.

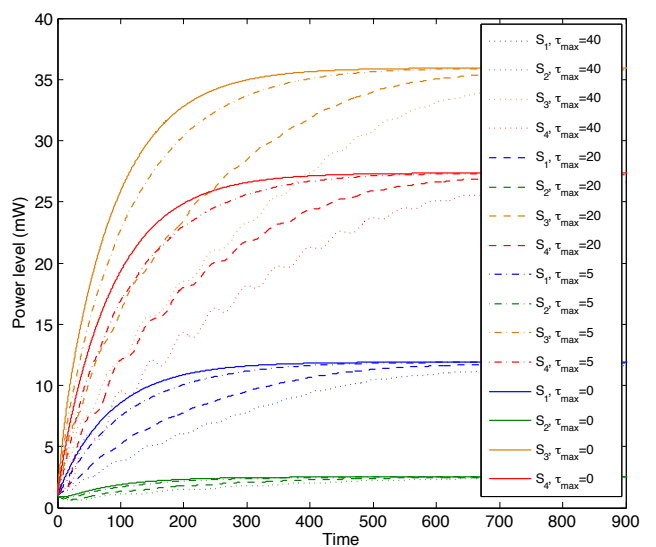

Fig. 1. Convergence of the UBPC algorithm with desired SINR $\gamma_{i}=1.5$, thermal noise $\eta=0.04 \mathrm{mWatts}$ and proportionality gain $k_{i}=0.1$ for all users. The solid, dashed, dot-dashed and dotted lines illustrate the convergence of the algorithm in the presence of delays with maximum delay $0,5,20$ and 40 , respectively.

The theoretical upper bounds on the decay rate of the UBPC algorithm as obtained in Theorem 2 have been compared with the actual decay rate of the UBPC algorithm 
for the wireless network characterized by the matrix (9), see Figure 2. Since the delays are time-varying and usually smaller than the maximum, there is a gap between the theoretical and the actual decay rates that one observes in simulations. As we have already shown, when delays are introduced, $\bar{c}$ increases monotonically with $\tau_{\max }$, meaning that the decay rate decreases. For this network, we have numerically calculated $\bar{c}$ for the values of delays we consider in the simulations and we have $\bar{c}_{5}=0.9193, \bar{c}_{20}=0.9588$ and $\bar{c}_{40}=0.9752$.

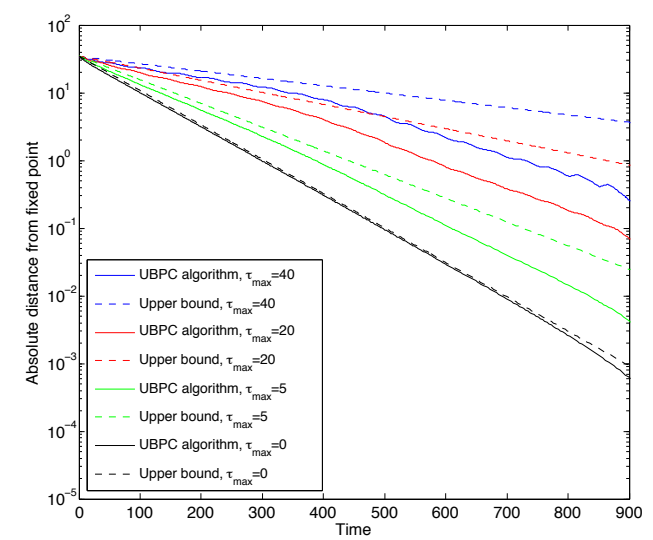

Fig. 2. Comparison of upper bound on the decay rate of the UBPC algorithm and the actual decay rate of the UBPC algorithm for the wireless network characterized by matrix (9), for $\tau_{\max }=5,20$ and 40 . The dashed lines show the theoretical upper bound on the decay rate, while the solid lines show the actual decay rate of the UBPC algorithm.

\section{CONCLUSIONS}

This paper developed a comprehensive stability analysis framework for continuous-time power control laws under bounded time-varying communication delays. The first set of results established global asymptotic stability of power control laws involving two-sided scalable interference functions. Next, we proved global exponential stability of power control laws involving contractive interference functions and derived explicit bounds on the decay rate which allows to, for the first time, quantify the impact of delays on the convergence rate of the transmit powers.

\section{APPENDIX}

\section{A. Proof of Theorem 1}

First note that if $\boldsymbol{p}^{\star}$ is a fixed point of the two-sided scalable interference function $\boldsymbol{I}(\boldsymbol{p})$, then $\boldsymbol{p}^{\star}=\boldsymbol{I}\left(\boldsymbol{p}^{\star}\right)>\mathbf{0}$. Since the initial condition $\varphi(\cdot)$ is assumed to be positive $(\varphi(\cdot)>0)$ and $\boldsymbol{I}(\boldsymbol{p})>\mathbf{0}$ for all $\boldsymbol{p} \geq \mathbf{0}$, the solutions of the positive system (3) satisfy $\boldsymbol{p}(t)>\mathbf{0}$ for all $t \geq 0[14$, Chapter 3]. This allows us to use the Lyapunov function

$$
V(\boldsymbol{p})=d\left(\boldsymbol{p}, \boldsymbol{p}^{\star}\right)-1
$$

where $d(\cdot, \cdot)$ is defined as in (5). Let $m$ be the index for which the maximum is achieved at time $t$. That is,

$$
m=\underset{1 \leq i \leq n}{\arg \max }\left\{\max \left\{\frac{p_{i}(t)}{p_{i}^{\star}}, \frac{p_{i}^{\star}}{p_{i}(t)}\right\}\right\} .
$$

This implies that

$$
V(\boldsymbol{p})=\max \left\{\frac{p_{m}(t)}{p_{m}^{\star}}, \frac{p_{m}^{\star}}{p_{m}(t)}\right\}-1 .
$$

It is clear that $V(\boldsymbol{p})>0$ for all $\boldsymbol{p} \neq \boldsymbol{p}^{\star}$. The time-derivative of the candidate Lyapunov function along the trajectories of (3) is given by

$$
\dot{V}(\boldsymbol{p})= \begin{cases}\frac{\dot{p}_{m}(t)}{p_{m}^{\star}}, & \text { if } p_{m}(t)>p_{m}^{\star}, \\ 0, & \text { if } p_{m}(t)=p_{m}^{\star}, \\ -\frac{p_{m}^{\star}}{p_{m}^{2}(t)} \dot{p}_{m}(t), & \text { if } p_{m}(t)<p_{m}^{\star} .\end{cases}
$$

a) If $p_{m}(t)>p_{m}^{\star}$, then

$$
\begin{aligned}
\dot{V}(\boldsymbol{p}) & =k_{m}\left(-\frac{p_{m}(t)+I_{m}\left(\boldsymbol{p}^{d_{m}}(t)\right)}{p_{m}^{\star}}\right) \\
& =k_{m}\left(-\frac{p_{m}(t)}{p_{m}^{\star}}+\frac{I_{m}\left(\boldsymbol{p}^{d_{m}}(t)\right)}{I_{m}\left(\boldsymbol{p}^{\star}\right)}\right),
\end{aligned}
$$

where we used the fact that $p_{m}^{\star}=I_{m}\left(\boldsymbol{p}^{\star}\right)$ to get the second equality. Since $d\left(\boldsymbol{p}(t), \boldsymbol{p}^{\star}\right)=\frac{p_{m}(t)}{p_{m}^{\star}}$, and

$$
\frac{I_{m}\left(\boldsymbol{p}^{d_{m}}(t)\right)}{I_{m}\left(\boldsymbol{p}^{\star}\right)} \leq d\left(\boldsymbol{I}\left(\boldsymbol{p}^{d_{m}}(t)\right), \boldsymbol{I}\left(\boldsymbol{p}^{\star}\right)\right),
$$

we have

$$
\dot{V}(\boldsymbol{p}) \leq k_{m}\left(-d\left(\boldsymbol{p}(t), \boldsymbol{p}^{\star}\right)+d\left(\boldsymbol{I}\left(\boldsymbol{p}^{d_{m}}(t)\right), \boldsymbol{I}\left(\boldsymbol{p}^{\star}\right)\right)\right) .
$$

On the other hand, by Proposition 2(c), we obtain

$$
\begin{aligned}
d\left(\boldsymbol{I}\left(\boldsymbol{p}^{d_{m}}(t)\right), \boldsymbol{I}\left(\boldsymbol{p}^{\star}\right)\right) \\
\quad<d\left(\boldsymbol{p}^{d_{m}}(t), \boldsymbol{p}^{\star}\right) \\
\quad=\max _{1 \leq i \leq n}\left\{\max \left\{\frac{p_{i}\left(t-\tau_{i}^{m}(t)\right)}{p_{i}^{\star}}, \frac{p_{i}^{\star}}{p_{i}\left(t-\tau_{i}^{m}(t)\right)}\right\}\right\} \\
\leq \max _{1 \leq i \leq n} d\left(\boldsymbol{p}\left(t-\tau_{i}^{m}(t)\right), \boldsymbol{p}^{\star}\right) \\
\quad \leq \max _{t-\tau_{\max } \leq s \leq t} d\left(\boldsymbol{p}(s), \boldsymbol{p}^{\star}\right),
\end{aligned}
$$

where the last inequality follows from the fact that $\tau_{i}^{m}(t) \leq \tau_{\max }$. Combining (10) and (11) gives

$$
\begin{aligned}
\dot{V}(\boldsymbol{p}) & <k_{m}\left(-d\left(\boldsymbol{p}(t), \boldsymbol{p}^{\star}\right)+\max _{t-\tau_{\max } \leq s \leq t} d\left(\boldsymbol{p}(s), \boldsymbol{p}^{\star}\right)\right) \\
& =k_{m}\left(-V(\boldsymbol{p}(t))+\max _{t-\tau_{\max } \leq s \leq t} V(\boldsymbol{p}(s))\right) .
\end{aligned}
$$

b) Similarly, if $p_{m}(t)<p_{m}^{\star}$, we have

$$
\begin{aligned}
\dot{V}(\boldsymbol{p}) & \leq k_{m} \frac{I_{m}(\boldsymbol{p}(t))}{p_{m}(t)}\left(-d\left(\boldsymbol{p}(t), \boldsymbol{p}^{\star}\right)+d\left(\boldsymbol{I}\left(\boldsymbol{p}^{d_{m}}(t)\right), \boldsymbol{I}\left(\boldsymbol{p}^{\star}\right)\right)\right) \\
& <k_{m} \frac{I_{m}(\boldsymbol{p}(t))}{p_{m}(t)}\left(-V(\boldsymbol{p}(t))+\max _{t-\tau_{\max } \leq s \leq t} V(\boldsymbol{p}(s))\right) .
\end{aligned}
$$

In order to address the asymptotic stability of the continuoustime power control algorithm (3), we now apply the Invariance-Principle theorem for delay differential equations [15, Corollary 1], [16, Corollary 3.3.2]. In the light of this theorem, we are interested in the case

$$
\max _{t-\tau_{\max } \leq s \leq t} V(\boldsymbol{p}(s))=V(\boldsymbol{p}(t)) .
$$


It follows from (12) and (13) that while the above condition holds, $\dot{V}(\boldsymbol{p})<0$ for all $\boldsymbol{p} \neq \boldsymbol{p}^{\star}$. Therefore, $\boldsymbol{p}=\boldsymbol{p}^{\star}$ is globally asymptotically stable.

\section{B. Proof of Theorem 2}

We use the candidate Lyapunov function

$$
V(\boldsymbol{p})=\left\|\boldsymbol{p}-\boldsymbol{p}^{\star}\right\|_{\infty}^{\boldsymbol{v}}=\max _{1 \leq i \leq n} \frac{1}{v_{i}}\left|p_{i}-p_{i}^{\star}\right| .
$$

Let $m$ be the index for which the maximum is achieved at time $t$. Calculating the time derivative of $V(\boldsymbol{p})$ along the solutions of (3), we get

$$
\begin{aligned}
& \dot{V}(\boldsymbol{p})=\frac{1}{v_{m}} \operatorname{sgn}\left(p_{m}(t)-p_{m}^{\star}\right) \dot{p}_{m}(t) \\
&=\frac{1}{v_{m}} \operatorname{sgn}\left(p_{m}(t)-p_{m}^{\star}\right) k_{m}\left(-p_{m}(t)+I_{m}\left(\boldsymbol{p}^{d_{m}}(t)\right)\right) \\
&=k_{m}\left(-\frac{1}{v_{m}} \operatorname{sgn}\left(p_{m}(t)-p_{m}^{\star}\right)\left(p_{m}(t)-p_{m}^{\star}\right)\right) \\
&+k_{m}\left(\frac{1}{v_{m}} \operatorname{sgn}\left(p_{m}(t)-p_{m}^{\star}\right)\left(I_{m}\left(\boldsymbol{p}^{d_{m}}(t)\right)-I_{m}\left(\boldsymbol{p}^{\star}\right)\right)\right) \\
& \leq k_{m}\left(-\frac{1}{v_{m}}\left|p_{m}(t)-p_{m}^{\star}\right|+\right. \\
&\left.\frac{1}{v_{m}}\left|I_{m}\left(\boldsymbol{p}^{d_{m}}(t)\right)-I_{m}\left(\boldsymbol{p}^{\star}\right)\right|\right),
\end{aligned}
$$

where we used the fact that $p_{m}^{\star}=I_{m}\left(\boldsymbol{p}^{\star}\right)$ to obtain the third equality. The interference function $\boldsymbol{I}(\boldsymbol{p})$ is c-contractive. Thus,

$$
\begin{aligned}
\frac{1}{v_{m}}\left|I_{m}\left(\boldsymbol{p}^{d_{m}}(t)\right)-I_{m}\left(\boldsymbol{p}^{\star}\right)\right| & \leq\left\|\boldsymbol{I}\left(\boldsymbol{p}^{d_{m}}(t)\right)-\boldsymbol{I}\left(\boldsymbol{p}^{\star}\right)\right\|_{\infty}^{\boldsymbol{v}} \\
& \leq c\left\|\boldsymbol{p}^{d_{m}}(t)-\boldsymbol{p}^{\star}\right\|_{\infty}^{\boldsymbol{v}}, \quad(1)
\end{aligned}
$$

where we used the definition of weighted $l_{\infty}$ norm to get the first inequality, and Proposition 3 to obtain the second inequality. Substituting (15) into (14) yields

$$
\begin{aligned}
\dot{V}(\boldsymbol{p}) & \leq k_{m}\left(-\left\|\boldsymbol{p}(t)-\boldsymbol{p}^{\star}\right\|_{\infty}^{\boldsymbol{v}}+c\left\|\boldsymbol{p}^{d_{m}}(t)-\boldsymbol{p}^{\star}\right\|_{\infty}^{\boldsymbol{v}}\right) \\
& =k_{m}\left(-\left\|\boldsymbol{p}(t)-\boldsymbol{p}^{\star}\right\|_{\infty}^{\boldsymbol{v}}+c \max _{1 \leq i \leq n} \frac{1}{v_{i}}\left|p_{i}\left(t-\tau_{i}^{m}(t)\right)-p_{i}^{\star}\right|\right) \\
& \leq k_{m}\left(-\left\|\boldsymbol{p}(t)-\boldsymbol{p}^{\star}\right\|_{\infty}^{\boldsymbol{v}}+c \max _{1 \leq i \leq n}\left\|\boldsymbol{p}\left(t-\tau_{i}^{m}(t)\right)-\boldsymbol{p}^{\star}\right\|_{\infty}^{\boldsymbol{v}}\right) \\
& =k_{m}\left(-V(\boldsymbol{p}(t))+c \max _{1 \leq i \leq n} V\left(\boldsymbol{p}\left(t-\tau_{i}^{m}(t)\right)\right)\right) \\
& \leq k_{m}\left(-V(\boldsymbol{p}(t))+c \max _{t-\tau_{\max } \leq s \leq t} V(\boldsymbol{p}(s))\right) .
\end{aligned}
$$

Since $V(\boldsymbol{p}) \geq 0$, by Halanay Inequality [17, pp. 389-390], we obtain

$$
V(\boldsymbol{p}(t)) \leq\left(\sup _{-\tau_{\max } \leq s \leq 0} V(\boldsymbol{p}(s))\right) e^{-\eta_{m} t}, \quad t \geq 0,
$$

where $\eta_{m}$ is the unique positive solution of the equation

$$
\eta_{m}=k_{m}\left(1-c e^{\eta_{m} \tau_{\max }}\right) \text {. }
$$

Let $\eta_{i}$ be the positive solution of the equation

$$
\eta_{i}=k_{i}\left(1-c e^{\eta_{i} \tau_{\max }}\right)
$$

for $i=1, \ldots, n$. Since $\eta_{i}$, as a function of $k_{i}>0$, is monotonically increasing, we have $\eta \leq \eta_{i}$ for all $i$ where

$$
\eta=k_{\min }\left(1-c e^{\eta \tau_{\max }}\right) .
$$

This implies that $\eta \leq \eta_{m}$, and hence $e^{-\eta_{m} t} \leq e^{-\eta t}$ for $t \geq 0$. Therefore,

$$
V(\boldsymbol{p}(t)) \leq\left(\sup _{-\tau_{\max } \leq s \leq 0} V(\boldsymbol{p}(s))\right) e^{-\eta t} .
$$

Letting $\bar{c}=1-\frac{\eta}{k_{\min }}$, the result follows immediately.

\section{REFERENCES}

[1] G. J. Foschini and Z. Miljanic, "A simple distributed autonomous power control algorithm and its convergence," IEEE Transactions on Vehicular Technology, vol. 42, pp. 641-646, 1993.

[2] M. M. Olama, S. M. Djouadi, and C. D. Charalambous, "A general framework for continuous time power control in time varying long term fading wireless networks," in Proceedings of the Ninth IASTED International Conference on Control and Applications, ser. CA '07. Anaheim, CA, USA: ACTA Press, 2007, pp. 69-74.

[3] W.-C. Su, B.-H. Jung, S.-Y. Chang, and Z. Gajic, "Unified continuousand discrete-time uplink power control problem formulation for SIRbased wireless networks," in IEEE Sarnoff Symposium, April 2009, pp. $1-5$.

[4] T. Charalambous, I. Lestas, and G. Vinnicombe, "On the stability of the Foschini-Miljanic algorithm with time-delays," in $C D C, 2008$, pp. 2991-2996.

[5] I. Lestas, "Power control in wireless networks: Stability and delay independence for a general class of distributed algorithms," IEEE Transactions on Automatic Control, vol. 57, no. 5, pp. 1253-1258, May 2012.

[6] A. Zappavigna, T. Charalambous, and F. Knorn, "Unconditional stability of the Foschini-Miljanic algorithm," Automatica, vol. 48, no. 1, pp. 219-224, 2012.

[7] R. Yates, "A framework for uplink power control in cellular radio systems," IEEE Journal on Selected Areas in Communications, vol. 13 , no. 7, pp. 1341-1347, 1995.

[8] C. Sung and K. Leung, "A generalized framework for distributed power control in wireless networks," IEEE Transactions on Information Theory, vol. 51, no. 7, pp. 2625-2635, 2005.

[9] A. Moller and U. Jonsson, "Input Output Analysis of Power Control in Wireless Networks," in Proceedings of the $49^{\text {th }}$ IEEE Conference on Decision and Control, December 2010, pp. 6451-6456.

[10] H. Boche and M. Schubert, "A unifying approach to interference modeling for wireless networks," IEEE Transactions on Signal Processing, vol. 58, no. 6, pp. 3282-3297, June 2010.

[11] H. Feyzmahdavian, M. Johansson, and T. Charalambous, "Contractive interference functions and rates of convergence of distributed power control laws," IEEE Transactions on Wireless Communications, vol. 11, no. 12, pp. 4494-4502, Dec. 2012.

[12] M. Xiao, N. Shroff, and E. Chong, "A utility-based power-control scheme in wireless cellular systems," IEEE Journal on Selected Areas in Communications, vol. 11, no. 2, pp. 210-221, 2003.

[13] D. P. Bertsekas and J. N. Tsitsiklis, Parallel and Distributed Computation. Prentice-Hall, 1989.

[14] W. M. Haddad, V. Chellaboina, and Q. Hui, Nonnegative and Compartmental Dynamical Systems. Princton, New Jersey, 2010.

[15] J. R. Haddock and J. Terjeki, "Lyapunov-Razumikhin functions and invariance principle for functional differential equations," Journal of Differential Equations, vol. 48, pp. 95-122, 1983.

[16] E. N. Chukwu, Stability and Time-Optimal Control of Hereditary Systems. Mathematics in Science and Engineering, Academic Press, 1992.

[17] R. D. Driver, Ordinary and Delay Differential Equations. Springer, New York, 1977. 